\title{
Degradation of Power Bipolar Operational Amplifiers in a Mixed Neutron \& Gamma Environment
}

\author{
F. J. Franco, Y. Zong and J. A. Agapito
}

\begin{abstract}
Power operational amplifiers were irradiated in a mixed neutron and gamma radiation environment. These experiments showed that the degradation of the power operational amplifiers shares a great deal of characteristics with that of the low signal devices (e. g., shift of the input offset voltage, increase of the input bias currents, and degradation of the frequency behavior). However, other phenomena were observed without equivalence in the family (linear dependence of the inverse of the quiescent current on the neutron fluence, more significant degradation of the negative output current and collapse of the primary operational amplifiers). These phenomena were explained from the special characteristics of the output stage, optimized to provide a current of several amperes. Finally, even though power devices are especially sensitive to radiation damage, some of the tested devices are suitable for radiation levels on the order of $5 \cdot 10^{13}-10^{14} \mathrm{n} \cdot \mathrm{cm}^{-2}$ in case the whole electronic system, in which the device is integrated, is carefully designed.
\end{abstract}

Index Terms-Bipolar technology, neutron damage, operational amplifiers, power devices, radiation tolerance.

\section{INTRODUCTION}

$\mathbf{P}$ OWER operational amplifiers (power op amps) make up a family of operational amplifiers that have been specially developed to provide a very high output current (on the order of several amperes) and also to deal with very high values of power supplies (some tens or even a hundred of volts). On the contrary, typical operational amplifiers usually cannot work with dual power supplies higher than $\pm 18 \mathrm{~V}$ and hardly provide some tens of milliamperes. To manage such large power supplies and currents, some improvements are added that unfortunately bring some side-effects such as a high output noise level or a large quiescent current to correctly work.

Like the rest of electronic devices, power op amps are liable to undergo degradation if they are exposed to radiation. Indeed,

Manuscript received...

This work was supported by the cooperation agreement K476/LHC between CERN \& UCM, by the Spanish Research Agency CICYT (FPA2002-00912), by the Spanish Agency for the International Cooperation (AECI) and by the "Miguel Casado San José" Foundation.

Francisco J. Franco is with the TIMA Laboratory, 46, Felix Viallet, 38031 Grenoble (France), on leave from the Departamento de Física Aplicada III, Facultad de Ciencias Físicas, Universidad Complutense de Madrid, 28040 Madrid (Spain). E-mail: monti@fis.ucm.es

Yi Zong is with the Departamento de Física Aplicada III, Facultad de Ciencias Físicas, Universidad Complutense de Madrid, 28040 Madrid (Spain), on leave from the Wuhan Institute of Technology, Wuhan (China). E-mail: yzong@fis.ucm.es.

Juan A. Agapito is with is with the Departamento de Física Aplicada III, Facultad de Ciencias Físicas, Universidad Complutense de Madrid, 28040 Madrid (Spain). E-mail: agapito@fis.ucm.es they are supposed to be very sensitive due to the fact that the family of the power transistors is one of the less tolerant to the radiation damage [1]. Surprisingly, in spite of being easy to gather data and discussions concerning the low signal operational amplifiers, whether in the scientific publications [2]-[23] or in public databases [24], it is really difficult to find data concerning the subset of power devices. To the authors' knowledge, the only available data were reported by Sharma et al. [25], O'Bryan et al. [26] and the GSFC public database [27], all of them concerning some devices from Apex Microtechnology. We hope that this paper will be helpful to compensate this scarcity of data.

However, this is not the only goal that has led to the research of the degradation of the power operational amplifiers. There is also a more practical reason, related to the development of a new particle accelerator, the Large Hadron Collider (LHC), nowadays being built at the European Organization for Nuclear Research (CERN) at Geneva (Switzerland). The cryogenic system of this huge nuclear research facility must use power operational amplifiers, which ought to be tolerant to the radiation created by the particle beams. This fact has yielded to two important consequences in the performance of the radiation tests: First of all, the radiation environment should simulate the LHC radiation environment and, also, the radiation tests must deeply investigate the evolution of the devices that showed the most promising behavior during a preliminary experiment.

\section{TEST SET-UP}

The experiment was scheduled to be performed in two steps. First, one only sample of different devices was irradiated with the goal of selecting the most tolerant amplifier (or amplifiers). Afterwards, a new irradiation on a larger set of samples of those promising devices was carried out to account for the preliminary results. For the first campaign, the following devices were selected: OPA541, from Texas Instruments and the PA10, PA12A \& PA61, from Apex Microtechnology [28], [29].

All the devices have a bipolar input stage except the OPA541, with a JFET input stage. However, the most important detail concerning the internal structure of the operational amplifiers is the nature of the output stage. Some characteristics shared by all of them are the presence of Darlington transistor pairs, in order to increase the size of the short circuit current, and the use of output stages other than the 


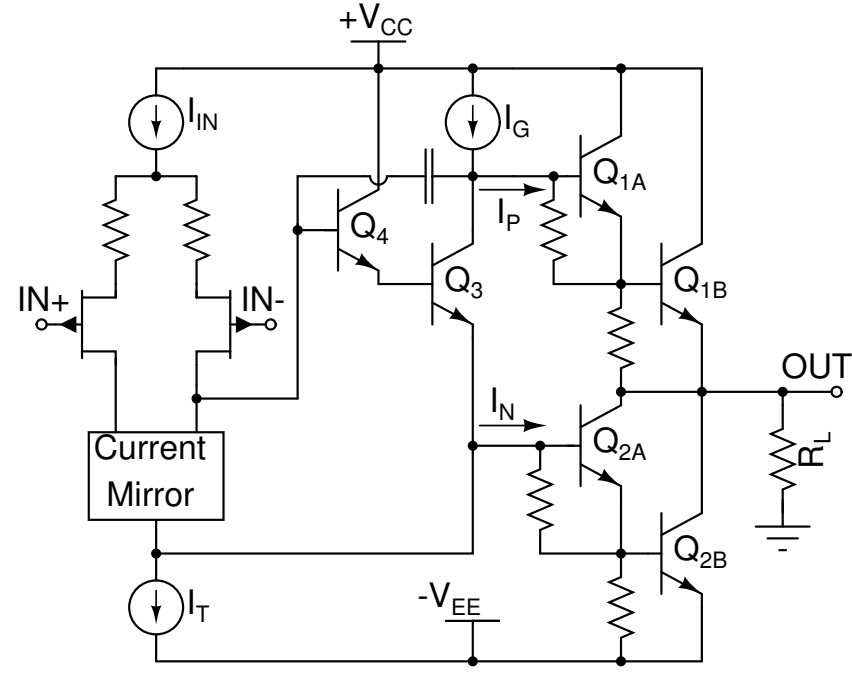

Fig. 1. OPA541's simplified internal structure. $Q_{2}$ and $Q_{3}$ along with $I_{G}$ make up the gain stage of the device. A fraction of $I_{G}\left(I_{P}\right)$ is deviated to $Q_{1 A}$ so that they be in forward-active zone. Thus, $V_{O U T}=$ $V_{G}-\left(V_{B E, Q 1 A}+V_{B E, Q 1 B}\right)$, extremely linear, but with the penalty of a continuous high power consumption.

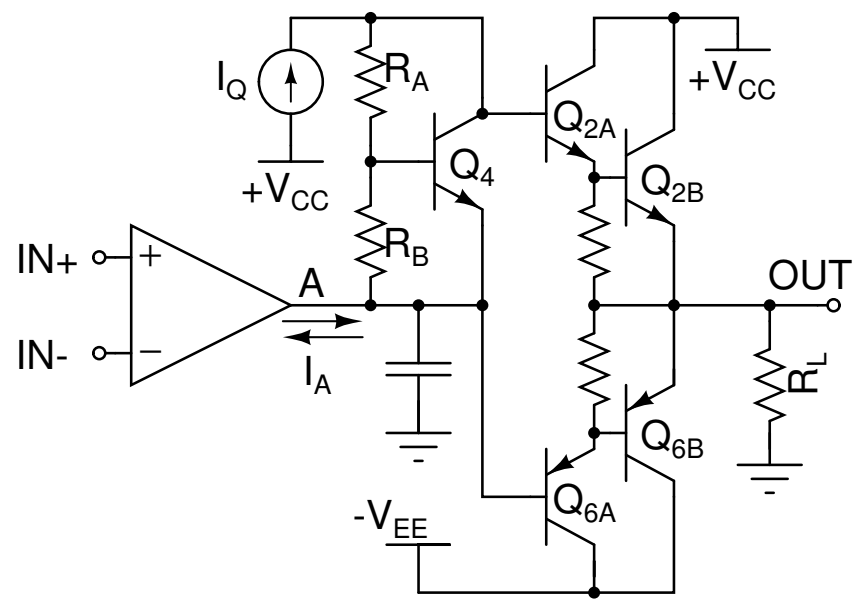

Fig. 2. PA1X's internal structure. In this structure, $Q_{2 X}$ is devoted to providing the output current whereas $Q_{6 X}$ must absorb it if necessary. In this structure, $V_{O U T}=V_{A}+\left(V_{B E, 6 A}+V_{B E, 6 B}\right)$ and, to guarantee the linearity of the stage, $R_{A} \approx 3 \cdot R_{B}$.

typical class $A B$ stage found in low signal devices [22]. In fact, the output structure is class A in OPA541 and C in PA61, the PA1X devices being class AB amplifiers (Figs. 13). Another important feature is that the manufacturer has added some special inputs to attach resistors with the goal of controlling the output current and protecting a hypothetical load. During the tests, these pins were shorted in order to remove any kind of limitation to the devices. In this context, some additional transistors present inside the device are led to cut-off state so they have been withdrawn from the initial schematics furnished by the manufacturers (Figs. 1-3).

The devices were irradiated at the neutron source of the Portuguese Research Reactor [30], which was especially designed to simulate the LHC radiation environment. In this facility, a neutron fluence similar to that of a 10-year LHC activity period

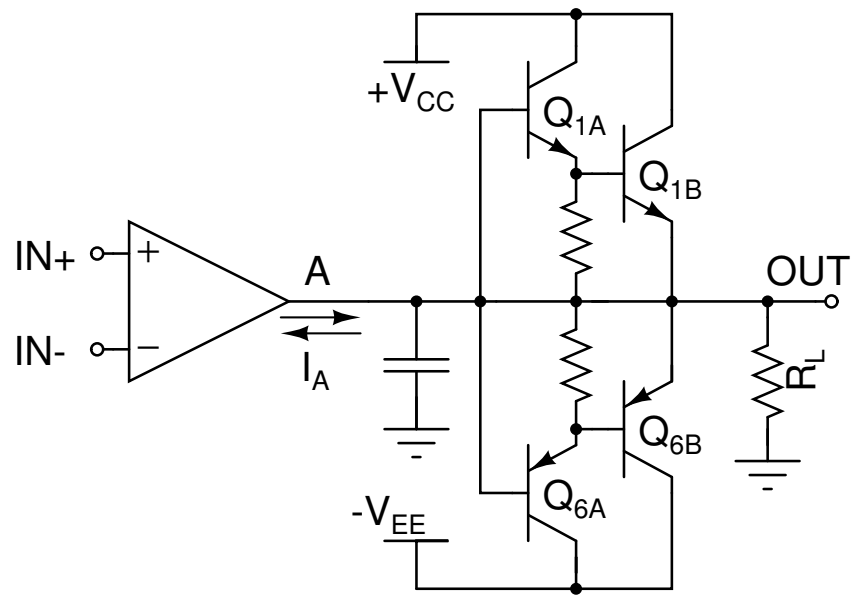

Fig. 3. PA61's internal structure. In this class $\mathrm{C}$ stage, $Q_{1 X}$ provides the output current whereas $Q_{6 X}$ absorbs it. This structure only works if $V_{A} \geq$ $2 \cdot V_{B E}, V_{B E}$ being the voltage drop along a forward biased PN junction $(\approx 0.7 \mathrm{~V})$. Thus, it may distort the output signal but minimizes the power consumption.

$\left(\sim 5 \cdot 10^{13} 1-\mathrm{MeV} \mathrm{n} \cdot \mathrm{cm}^{-2}\right)$ is reached after $60 \mathrm{~h}$ of irradiation. Moreover, the total ionizing dose (TID) is on the order of 2$2.5 \mathrm{kGy}(\mathrm{Si})$ (Dose Rate $\sim 40 \mathrm{~Gy} / \mathrm{h}$ ). The neutron fluence was measured with ${ }^{58} \mathrm{Ni}$ foils and converted into standard $1-\mathrm{MeV}$ $\mathrm{n} \cdot \mathrm{cm}^{-2}$ multiplying the measured value by 1.28 , as previous calculations have shown [31]. In addition, the total ionizing dose continuously tracked with an ionization chamber.

According to the ATLAS radiation test protocol [32], in the case of neutron irradiations, the devices ought to be irradiated with all of the pins shorted. However, we preferred to test the devices in a similar situation to that in which the devices are supposed to work since, by this way, we could register an almost continuous evolution of some parameters such as the input offset voltage, the closed loop gain, etc. Therefore, we prepared the experiments so that the devices should be permanently biased and providing a very high current every ten minutes. The samples were biased as unity-gain buffers with $\pm 15 \mathrm{~V}$ power supplies and loaded with a $5.1 \Omega$-resistor (Fig. 4). Cyclically, a personal computer connected the input to a voltage source where a sweep between $\pm 5 \mathrm{~V}$ with a 0.2 $\mathrm{V}$ step was immediately done. Thus, the amplifier was forced to provide and absorb currents on the order of $1 \mathrm{~A}$, in any case for no longer than $25 \mathrm{~s}$. Finally, all of the pairs of input and output voltages were registered by the computer for a later careful examination.

Dealing with such high currents and voltages leads to a great power dissipation. In order to avoid the overheating of the devices, a cooling system injected cold air without interruption inside the cavity. Also, the temperature was measured by the personal computer with the help of four PT-100 resistors distributed along the longitudinal cavity axis. According to the computer log, the room temperature never was higher than 40 $\mathrm{C}$ at the inner side of the cavity, where the hot air drain was less efficient.

During the irradiation, parameters such as the highest output currents could be measured in the case of a drop below $1 \mathrm{~A}$. 


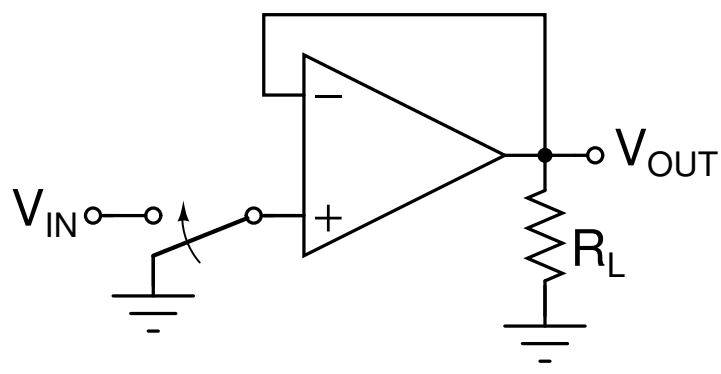

Fig. 4. Feedback network during the irradiation. The switch (a mechanical relay immune to radiation) was controlled by a program developed in Testpoint@ running in a personal computer. The value of $R_{L}$ is $5.1 \Omega$.

Also, the system could measure the input offset voltage, $V_{O S}$, as well as the variations of the closed loop gain. Input and output voltages were measured with an $8 \frac{1}{2}$-digit multimeter by means of a very low impedance cable inside a shielded pipe. This shield along with the absence of ground loops in the system allowed measuring those voltages with a high accuracy notwithstanding that the instrumentation system was five meters far away from the neutron facility.

After the irradiation, the rest of operational amplifier parameters, such as the open loop gain, the slew rate, etc., were measured once the vanishing of radioactive isotopes allowed a safe handling of the devices.

Finally, it is important to remark that in a preliminary experiment we tested all of the previously listed devices, from which we realized that the OPA541 was the most tolerant to the radiation. Therefore, a second round was performed irradiating a larger set of samples, coming from different batches and also having different packages (Power plastic and TO-3). This fact explains the abundance of data of this device in comparison to the others.

\section{RESUlts}

\section{A. OPA541}

The samples of this device received a total radiation dose between $1.02 \cdot 10^{14} 1-\mathrm{MeV} \mathrm{n} \cdot \mathrm{cm}^{-2} \& 2680 \mathrm{~Gy}(\mathrm{Si})$ and $2.6 \cdot 10^{13} 1-\mathrm{MeV} \mathrm{n} \cdot \mathrm{cm}^{-2} \& 1510 \mathrm{~Gy}(\mathrm{Si})$, the exact values being reported in Table I. Regarding the evolution of their main parameters, it was clear that the evolution of this device was not very different from the typical one of the low signal devices. In fact, variations of the input offset voltage were observed (Fig. 5), making this parameter randomly shift between $\pm 10 \mathrm{mV}$ without a clear relationship between the final value and the neutron fluence. Actually, some samples seem to be more damaged with low values of the neutron fluence than that at the end of the irradiation. Closely related to this parameter, the value of the PSRR also increased although, just like the input offset voltage, its evolution seems to follow a random pattern.

The value of the input bias current also increased but the final value was never higher than $1 \mathrm{nA}$. Also, we observed a decrease of the open loop gain, the gain-bandwidth product and slew rate, the most important damage observed in the slew rate (Fig. 6-7).

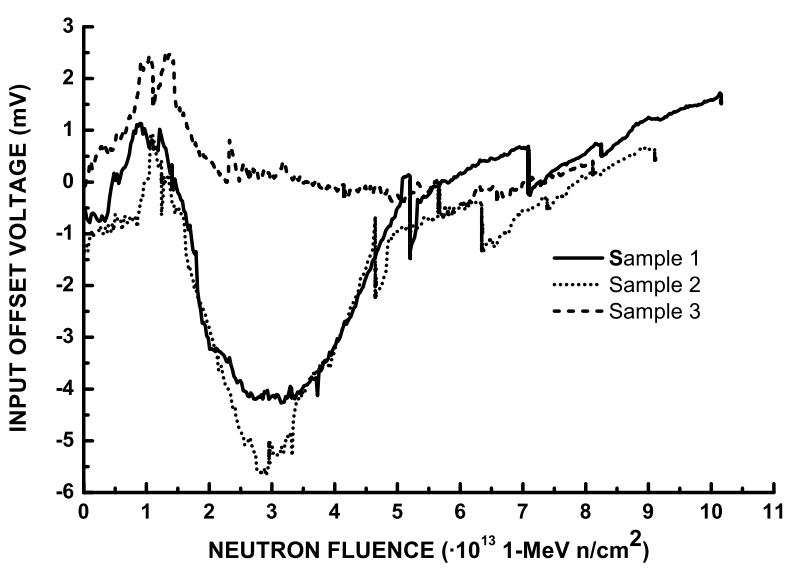

Fig. 5. Evolution of the input offset voltage of the three most irradiated samples of the OPA541

TABLE I

TOtAL RADIATION DOSE RECEIVED BY THE SAMPLES OF THE OPA54 1 DURING THE SECOND IRRADIATION.

\begin{tabular}{ccccc}
\hline Sample & Package & Neutron Fluence & TID & Dose Rate \\
\hline 1 & TO-3 & 10.2 & 2680 & 44.7 \\
\hline 2 & Plastic & 9.1 & 2540 & 42.3 \\
\hline 3 & TO-3 & 8.1 & 2400 & 40.0 \\
\hline 4 & Plastic & 7.2 & 2270 & 37.9 \\
\hline 5 & TO-3 & 5.6 & 2040 & 34.0 \\
\hline 6 & Plastic & 4.9 & 1940 & 32.3 \\
\hline 7 & TO-3 & 2.9 & 1590 & 26.4 \\
\hline 8 & Plastic & 2.6 & 1510 & 25.2 \\
\hline & & $\cdot 10^{\mathrm{I3}} \mathrm{n} \cdot \mathrm{cm}^{-2}$ & $\mathrm{~Gy}(\mathrm{Si})$ & $\mathrm{Gy}(\mathrm{Si}) / \mathrm{h}$ \\
\hline
\end{tabular}

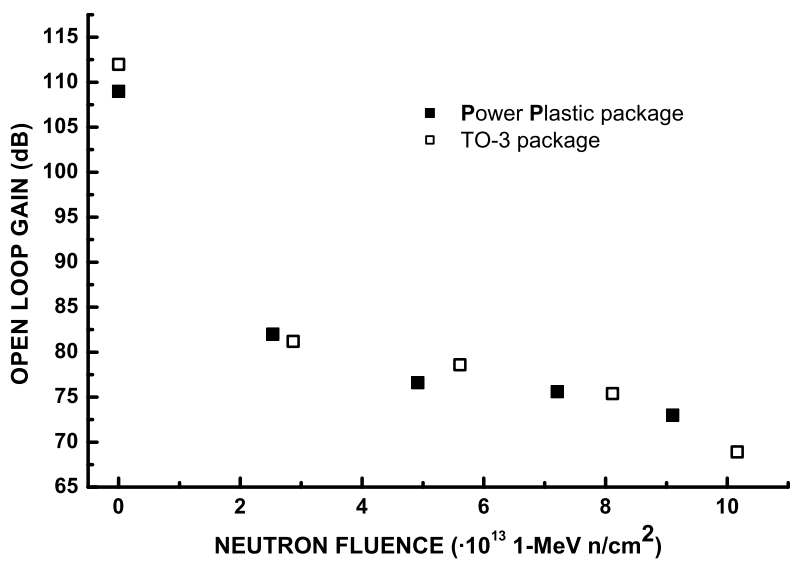

Fig. 6. Evolution of the open loop gain in the OPA541. 


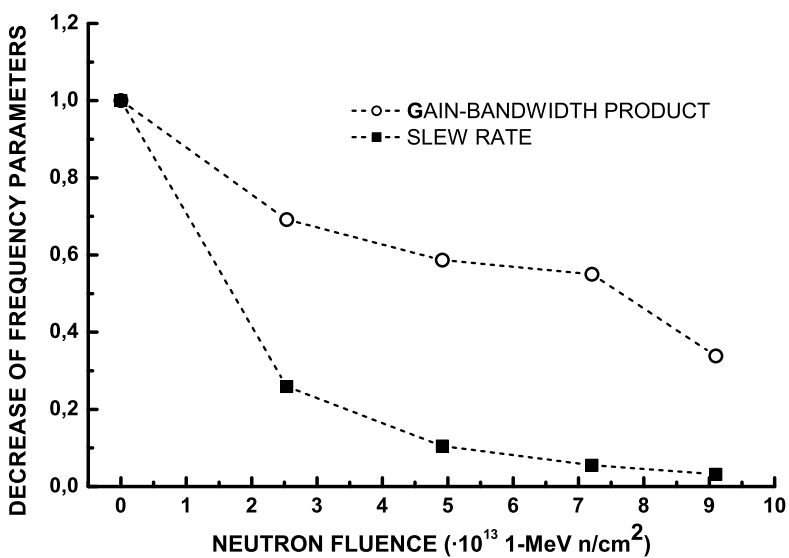

Fig. 7. Ratio between the final and initial values of the gain-bandwidth product and the slew rate to show the worsening of the frequency behavior of the OPA541AP. The initial values of slew rate and gain-bandwidth product were $12 \mathrm{~V} / \mu$ s and $2.4 \mathrm{MHz}$.

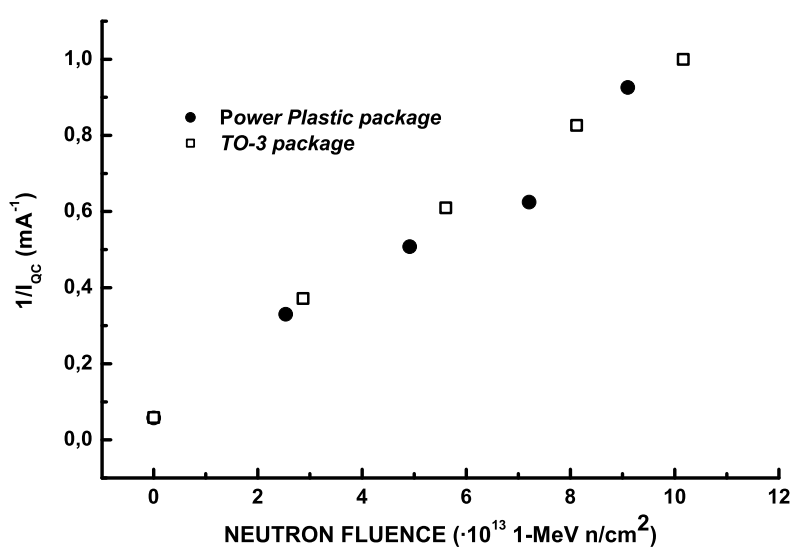

Fig. 8. Evolution of the OPA541's quiescent current in proportion to the neutron fluence.

In any case, the most interesting behavior concerns the evolution of the quiescent current (current provided by the power supplies while the devices were not loaded), $I_{Q C}$. In this device, there was a linear dependence between the values of $I_{Q C}^{-1}$ and the neutron fluence (Fig. 8). Another interesting property is the anomalous difference between the behavior of the positive and the negative output current. In fact, notwithstanding that the amplifiers are not able to provide more than $1 \mathrm{~A}$ at $1.3-1.9 \cdot 10^{13} 1-\mathrm{MeV} \mathrm{n} \cdot \mathrm{cm}^{-2} \& 250-800$ Gy, they may absorb more than $1 \mathrm{~A}$ up to $9 \cdot 10^{13} 1-\mathrm{MeV} \mathrm{n}$. $\mathrm{cm}^{-2}$. As we will see later, this is unusual since in the rest of amplifiers the negative output current is more radiationsensitive than the positive one (Fig. 9).

\section{B. PA10 \& PA12A}

These devices are manufactured by Apex Microtechnology and they are very similar to each other with the only difference of a higher output current provided by the PA12A. A sample of the PA12A received $4.18 \cdot 10^{13} 1-\mathrm{MeV} \mathrm{n} \cdot \mathrm{cm}^{-2}$ and 1125

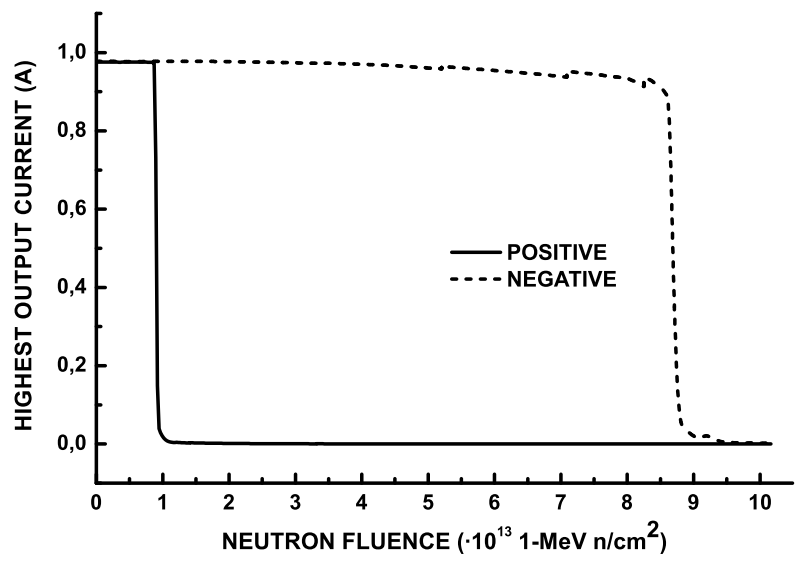

Fig. 9. Evolution of the highest output currents of the OPA541AM.

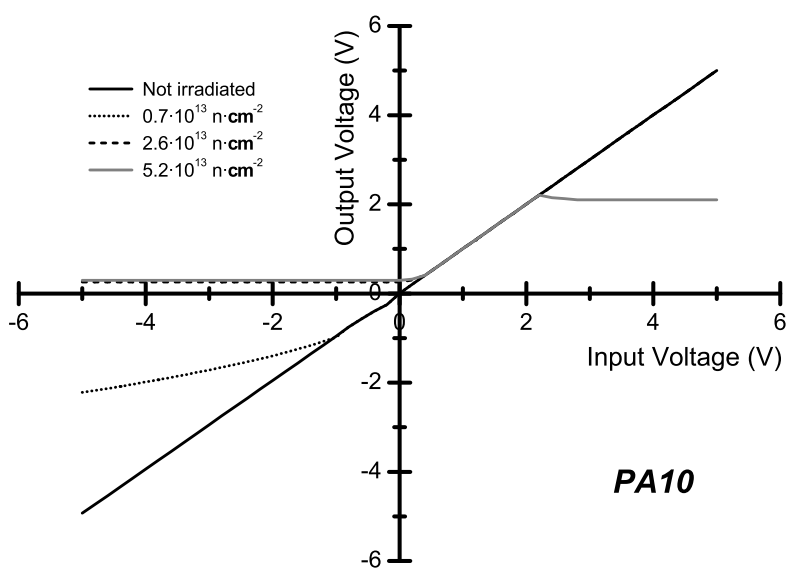

Fig. 10. DC input-output relationship of the PA10 with different values of neutron fluence.

Gy $(18.8 \mathrm{~Gy} / \mathrm{h})$ while the PA10, 5.96 $10^{13} 1-\mathrm{MeV} \mathrm{n} \cdot \mathrm{cm}^{-2}$ and $1220 \mathrm{~Gy}(20.3 \mathrm{~Gy} / \mathrm{h})$. None of them could work once the irradiation ended. In fact, the output of both devices was identical to the positive output saturation voltage even when the load was removed.

In this situation, only a small number of parameters could be measured. We observed an increase of the input bias current (on the order of $0.5 \mu \mathrm{A}$ ) and a decrease of the quiescent current (e.g., in the PA12A, $12 \mathrm{~mA} \rightarrow 3.8 \mathrm{~mA}$ ). Given this poor background, the only available data were those obtained from the on-line test, which showed a quick decrease of the negative output current and much slower in the case of the positive one. In fact, when the neutron fluence reached a value of $10^{13} 1$ $\mathrm{MeV} \mathrm{n} \cdot \mathrm{cm}^{-2}$, the devices' output voltage is restricted to the positive values, as if the devices were biased between 0 and $+15 \mathrm{~V}$ (Fig. 10). A consequence of this phenomenon is an apparent increase of the input offset voltage (Fig. 11). 


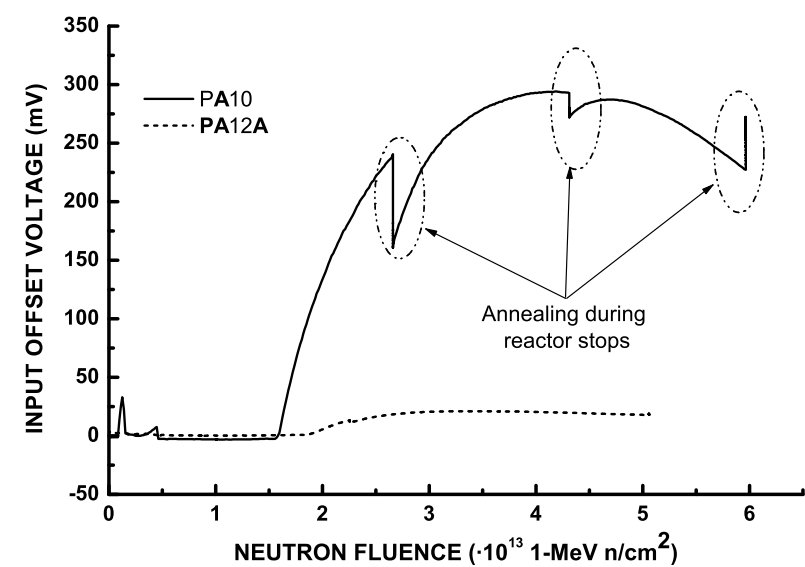

Fig. 11. Evolution of the PA1X's input offset voltage. The peaks present in the curve corresponding to the PA10 are related to the nocturnal annealing during the reactor stops. The origin of the $30-\mathrm{mV}$ spike at the beginning of the irradiation is unknown.

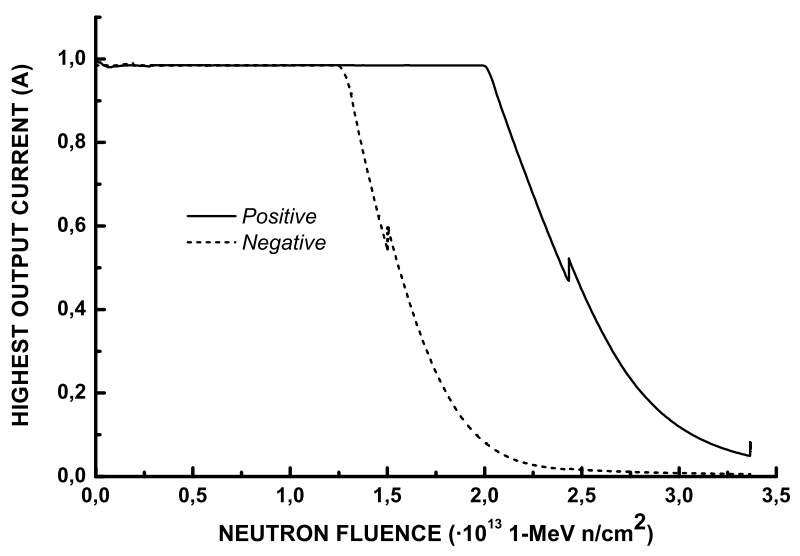

Fig. 12. Evolution of the PA61's highest output current, both positive and negative ones.

\section{PA61}

This amplifier, which received a total radiation dose of 3.36 . $10^{13} 1-\mathrm{MeV} \mathrm{n} \cdot \mathrm{cm}^{-2}$ and $870 \mathrm{~Gy}(14.5 \mathrm{~Gy} / \mathrm{h})$, showed a random shift in the input offset voltage during the irradiation, around $0 \mathrm{mV}$ but never beyond $2 \mathrm{mV}$. The measurement system also registered a steady decrease of the highest value of the output current, quicker in the case of the negative current (Fig. 12).

Although very damaged, the amplifier could work after the irradiation showing an input bias current of $300 \mathrm{nA}$ (former, $6 \mathrm{nA})$ and a lower quiescent current $(2.4 \mathrm{~mA} \rightarrow 1.6 \mathrm{~mA})$. Unfortunately, other parameters like the open loop gain or the frequency parameters could not be measured because of the very significant distortion of the output signal.

\section{Discussion}

The first feature deduced for the experimental results is that the degradation of the irradiated power operational amplifiers was quite similar to that observed in low signal devices. In fact, parallel evolutions in the typical parameters can be found in the related literature. Thus, it is widely accepted that the input offset voltage shifts with the radiation [4], [5], [7]-[9], [11], that the input bias currents always increase [5]-[10], [13], [21], that the open loop gain always falls, sometimes dramatically [3], [20], [21], etc. It is particularly interesting the evolution reported by Hiemstra concerning the OPA111's input offset voltage [15], which seems to show a random evolution similar to those observed in the devices tested in this paper. Besides, the lower tolerance of the slew rate to the radiation than that found in the gain-bandwidth product has been reported by the authors in low signal amplifiers tested in the same environment [23].

However, some phenomena seem to be specific of power operational amplifiers and, according to the authors' knowledge, have not been hitherto reported.

\section{A. Larger degradation of the PNP output transistors}

One of the typical degradations of irradiated bipolar operational amplifiers is the diminution of the output current (socalled short circuit current as well) due to the degradation of the transistors of the output stage. Moreover, this degradation may be accelerated by the degradation of the primary operational amplifier (Fig. 2-3), a goal of which is to inject or to absorb the highest possible current in the base of the Darlington pairs, which will be finally amplified and sent to the load.

Excluding the case of the OPA541, the degradation seems to be more important in the negative output current than in the positive one (e. g., Fig. 12). This behavior may be easily explained bearing in mind that, according to previous works (e. g. [1]), the PNP transistors are more sensitive to radiation than the NPN ones. There are two reasons to explain this behavior: First, let us imagine a PNP transistor derived from an NPN one just inverting the type of doping but keeping the geometry untouched. In this case, the current gain would be lower due to the smaller mobility of the holes, fact that accentuates the effect of the displacement damage. Also, it is necessary to highlight that a great deal of PNP transistors is built in lateral technology, with a lower tolerance to the displacement damage.

Thus, the quicker degradation of the PNP Darlington pair controlling the negative output current would explain that most of the amplifiers may provide a larger current than that they can absorb. However, the behavior of the OPA541 is really contrary to this trend. Indeed, the tolerance of the negative output current is seven times more than that of the positive one. To explain this anomalous behavior, it is necessary to return to Fig. 1 and realize that there is no PNP transistor in the output stage but only NPN transistors.

In this situation, is it possible to explain the different behavior between the positive and the negative currents of the OPA541? Unfortunately, only a deep knowledge of the internal structure of the amplifier would allow understanding the evolution. However, some hints would associate the accelerated degradation of the positive current with a likely higher 
sensitivity of the $Q_{1}$ pair. The reason of this supposition is that, checking the network of Fig. 1, the following condition arises:

$$
I_{P}+I_{N}=I_{I N}+I_{G}-I_{T}
$$

In this equation, the currents on the right side are constant since they are current sources. In other words, $I_{P}$ and $I_{N}$ are symmetric. When the amplifier must provide a high output current, $I_{P}$ increases at the expense of $I_{N}$, which drops down to 0 . On the other hand, if the amplifier must absorb the current instead of providing it, the roles are swapped. In conclusion, the bias current of both $Q_{1} \& Q_{2}$ pairs is the same in the situation of heavy loading so only a faster decrease of the $Q_{1}$ current gain could explain the quicker degradation of the positive output current.

\section{B. Linear dependence of $I_{Q C}^{-1}$ on the displacement damage}

A shocking result arisen from the experimental results is the existence of a clear linear relationship between $I_{Q C}^{-1}$ and the total neutron fluence, $\Phi$, in the case of the OPA541. On the contrary, if we represent this parameter as a function of the total ionizing dose, the dots are not so rightly distributed. In the past, some authors have reported a decrease in the power consumption of several general purpose operational amplifiers [16], [20] but never has it been found such a clear mathematical relation linking both parameters. Obviously, the fact that the OPA541 is a power device must be a key to understand such a predictable behavior.

One of the main drawbacks of the power operational amplifiers (excluding class $\mathrm{C}$ devices) is the high quiescent current, the most important contribution being that of the output stage, which usually needs a large current to be correctly biased [33].

Most of the low power operational amplifiers have a quiescent current in the order of 0.1-5 mA whereas operational amplifiers like the OPA541 have a typical quiescent current of 20-25 mA. Obviously, such a large current must be associated with the requirements of the output stage. Therefore, let us assume that the quiescent current of the whole operational amplifier can be approximated to that of the output stage $\left(I_{Q C} \approx I_{Q C, O U T}\right)$.

Checking Fig. 1, supposing $R_{L} \rightarrow \infty$ and realizing that the current flowing along the emitter of $Q_{1 B}$ is the same as the sum of those ones flowing through the collectors of $Q_{2 X}$, it is easy to demonstrate the following relation:

$$
I_{P} \cdot\left(1+h_{1 A}\right) \cdot\left(1+h_{1 B}\right)=I_{N} \cdot\left(1+h_{2 A}\right) \cdot\left(1+h_{2 B}\right)
$$

Combining this equation with (1) and accepting $h_{F E, X}>>$ 1 , the following relation arises:

$$
I_{P}=\frac{1}{1+\frac{h_{1 A} \cdot h_{1 B}}{h_{2 A} \cdot h_{2 B}}} \cdot \xi
$$

where $\xi=I_{N}+I_{P}-I_{T}$. Besides, accepting that the contribution of the input and gain stage is much less significant than that of the output stage and knowing that the power consumption of the output stage is just the current flowing through the collectors of the $Q_{1 X}$ Darlington pair, which integrally ends on the collectors of $Q_{2 X}$ :

$$
I_{Q C} \approx I_{Q C, O U T}=h_{F E, 1 A} \cdot h_{F E, 1 B} \cdot I_{P}
$$

Combining (3) and (4):

$$
I_{Q C}^{-1}=\xi^{-1} \cdot\left(\frac{1}{h_{F E, 1 A} \cdot h_{F E, 1 B}}+\frac{1}{h_{F E, 2 A} \cdot h_{F E, 2 B}}\right)
$$

However, the values of $h_{F E, X}^{-1}$ is ruled by the MessengerSpratt's law [1] so:

$$
\begin{aligned}
& I_{Q C}^{-1}= \\
& \xi^{-1} \cdot\left[\left(h_{F E, 1 A 0}^{-1}+K_{1 A} \cdot \Phi\right) \cdot\left(h_{F E, 1 B 0}^{-1}+K_{1 B} \cdot \Phi\right)\right. \\
& \left.\quad+\left(h_{F E, 2 A 0}^{-1}+K_{2 A} \cdot \Phi\right) \cdot\left(h_{F E, 2 B 0}^{-1}+K_{2 B} \cdot \Phi\right)\right]
\end{aligned}
$$

In this equation, we have supposed that the evolution of each transistor is different from the others' so there are four values of $K_{X Y}$. In other words, we have demonstrated that, in the case of the OPA541, there is a simple polynomial relationship between $I_{Q C}^{-1}$ and the neutron fluence.

However, this relationship may become linear accepting a well-known fact: Whether being exposed to ionizing radiation or displacement damage, the lower the base current, the more significant the diminution of the current gain, $h_{F E}$. Some examples of this behavior may be found in [2], [3] so it is possible that the current gain may become 0 in the range of low values of the base current while, in the range of the highest base currents values, the current gain has scarcely been affected. Now, let us remember that, in a Darlington pair, the base current of the second transistor is much higher than that of the first one. Therefore, the first transistor of the pair may be really degraded while the second one is softly damaged. In other words, $h_{F E, 1 B 0}^{-1}, h_{F E, 2 B 0}^{-1}>>K_{1 B} \cdot \Phi, K_{2 B} \cdot \Phi$. Thus, (6) becomes:

$$
\begin{aligned}
I_{Q C}^{-1}=\xi^{-1} \cdot\left[\left(h_{F E, 1 A 0}^{-1}+K_{1 A} \cdot \Phi\right) \cdot h_{F E, 1 B 0}^{-1}+\right. \\
\left.\left(h_{F E, 2 A 0}^{-1}+K_{2 A} \cdot \Phi\right) \cdot h_{F E, 2 B 0}^{-1}\right]
\end{aligned}
$$

and the linear relationship is finally deduced.

\section{Collapse of the primary op amp in PAIX}

Other astonishing phenomenon was the inability of PA1X to provide a negative output voltage. In other words, the DC input-output relationship becomes similar to that of an op amp biased with a unipolar power supply, the saturation voltage being some tenths of volt above the ground voltage (Fig. 10).

This phenomenon must not be associated with the incapacity of the PNP Darlington pair to absorb current. If so, the negative saturation voltage will get lower and lower, decreasing asymptotically to $0 \mathrm{~V}$. In fact, this is what SPICE simulations have shown and what we observed in other devices, e.g., the OPA541 when the positive output current fell down to $0 \mathrm{~A}$. 
Therefore, it is necessary to find a different reason of that behavior. In our opinion, it may be associated with the inability of the primary operational amplifier to bias the network of the output stage. In the structure of Fig. 2, positive output current is provided by an NPN pair, $Q_{2}$, whose base current comes from the current source, $I_{1}$, built with a Zener diode and resistors. On the contrary, if the output voltage is negative and the amplifier must absorb current, the active pair is $Q_{6}$, a PNP one. Obviously, in this situation, the operational amplifier is the device with the charge of extracting the PNP base current. Moreover, we must not forget that $I_{1}$ must be drained and the only path to return to the negative power supply is going into the power operational amplifier.

In short, the NPN Darlington pair is biased by a radiation tolerant current source whereas the PNP one is by an operational amplifier that, moreover, must absorb $I_{1}$ when this current source is not biasing the NPN transistors, this situation happening when there is a negative output voltage. Previous works [7], [22] have demonstrated that the op amps short circuit current is very affected by the radiation damage and may fall from values on the order of 20-40 mA down to a value lower than $1 \mathrm{~mA}$. In this situation, it will not be strange that the highest current that the op amp may deal with is lower than $I_{1}$.

Therefore, an anomalous situation comes out since no current is allowed to go out of the base of the PNP Darlington pair. In consequence, this fact drives the pair to cut-off state and to become no longer active. In consequence, the output stage loses the part dedicated to the absorption of current and, in practice, is converted into a class B stage able to provide but not to absorb current. Thus, it would be explained why the power operational amplifiers behave as if they were biased with only one power supply instead of two.

\section{Displacement damage vs. TID}

The last question to deal with is the role played by the gamma rays and the neutrons in the degradation of the devices since bipolar devices are quite sensitive to both kinds of radiation.

Some data point out a more significant contribution of the neutron radiation. Looking up in the GSFC database [27], some radiation tests summaries concerning PA10 and PA51 (an operational amplifier with the same output stage as the PA61) can be found. These documents report that, after a 1$\mathrm{kGy}{ }^{60} \mathrm{Co}$ gamma irradiation and $168 \mathrm{~h}$ of room temperature annealing, the most affected parameter was the input base currents. In fact, there is not any reference to any kind of phenomena similar to those depicted in this paper. On the contrary, the PA10 sample reported in this paper was useless with a TID value of $250 \mathrm{~Gy}$ (Fig. 11). The only way to explain this discrepancy is to accept that the main contribution was that of the neutrons.

However, in case the devices were irradiated with much higher TID values than those reached in our experiments, the internal transistor could undergo degradation similar to that caused by the neutrons. Therefore, the whole operational amplifier could show degradation not very different from that reported in this paper.

\section{CONClusion}

This paper has shown some interesting results concerning the degradation of power bipolar operational amplifiers irradiated in a mixed neutron \& gamma radiation. The results show that the evolution of this kind of devices seems to be similar to that observed in the family of the low power devices. Nevertheless, some phenomena seem to be exclusive of this family since they are related to the peculiar architecture of the output stage, which is the main difference between the low power and power families of the operational amplifiers.

Experimental data suggest that a complete electronic system may be hardened if some details are taken into account. For instance, in the case of the OPA541, given that the negative output current is less sensitive than the positive one, a key decision like using this device as a current sink rather than a source would increase the radiation tolerance of the total system. Finally, it is important to remark that, in spite of the fact that these phenomena were attributed to the displacement damage, similar behaviors would be observed in much harsher gamma environment since the phenomena are supposed to be related to typical degradations such as the diminution of the current gain.

\section{REFERENCES}

[1] G. C. Messenger and M. S. Ash, The Effects of Radiation on Electronic Systems, 2nd Edition, New York, Van Nostrand Reinhold, 1992.

[2] W. E. Combs, J. F. Krieg, P. L. Cole, and B. E. Eiche, "Radiation Effects on 73-E AT\&T CBIC LDEXR Bipolar Process," IEEE Radiation Effects Data Workshop, Tucson (USA), July 1994, pp. 110-117.

[3] O. Flament et al., "Radiation effects on SOI Analog Devices Parameters," IEEE Trans. Nucl. Sci., Vol. 41, no. 3, pp. 565-571, Jun. 1994.

[4] D. M. Hiemstra, "Dose Rate and Total Dose Noise Performance of a Commercial off the Shelf Dielectrically Isolated JFET Operational Amplifier during Irradiation," IEEE Radiation Effects Data Workshop, Indian Wells (USA), July 1996, pp. 1-5.

[5] C. I. Lee, B. G. Rax, and A. H. Johnston, "Total Dose Hardness Assurance Techniques for New Generation COTS Devices," IEEE Trans. Nucl. Sci., vol. 43, no. 6, pp. 3145-3150, Dec. 1996.

[6] R. L. Pease and M. Gehlhausen, "Elevated Temperature Irradiation of Bipolar Linear Microcircuits," IEEE Trans. Nucl. Sci., vol. 43, no. 6, pp. 3161-3168, Dec. 1996.

[7] L. Bonora and J. P. David, "An Attempt to Define Conservative Conditions for Total Dose Evaluation of Bipolar ICs," IEEE Trans. Nucl. Sci., Vol. 44, no. 6, pp. 1974-1980, Dec. 1997.

[8] C. I. Lee and A. H. Johnston, "Comparison of Total Dose Effects on Micropower Opamps: Bipolar and CMOS," IEEE Radiation Data Workshop, July, 24th 1998 , pp. 132-136.

[9] R. L. Pease et al., "Evaluation of Proposed Hardness Assurance Method for Bipolar Linear Circuits with Enhanced Low Dose Rate Sensitivity (ELDRS)," IEEE Trans. Nucl. Sci., vol. 45, no. 6, pp. 2665-2672, Dec. 1998.

[10] D. M. Hiemstra, "Dose Rate Dependence of the Current Noise Performance of an Ultra-Low Noise Precision Bipolar Operational Amplifier," IEEE Trans. Nucl. Sci., vol. 46, no. 6, pp. 1674-1679, Dec. 1999.

[11] R. L. Pease, J. Krieg, M. Gehlhausen, D. Platteter, and J. Black, "Total Dose Induced Increase in Input Offset Voltage in JFET Operational Amplifiers," Proc. of the Fifth European Conference on Radiation Effects on Components and Systems, Fontevraud (France), September 13-17 ${ }^{\text {th }}$, 1999, pp. 569-572.

[12] J. D. Saussine, D. Harmonic, E. Feuilloley, and O. Feuillatre, "Components Off the Shelf for Nuclear Industry Environment greater than 100 $\mathrm{kGy}(\mathrm{Si})$," Proc. of the Fifth European Conferences on Radiation Effects on Components and Systems, Fontevraud (France), September 13-17 ${ }^{\text {th }}$, 1999, pp. 424-428.

[13] H. Barnaby et al., "Origins of Total-Dose Response Variability in Linear Bipolar Microcircuits," IEEE Trans. Nucl. Sci., vol. 47, no. 6, pp. 23422349, Dec. 2000. 
[14] T. Carrière, R. Ecoffet, and P. Poirot, "Evaluation of Accelerated Total Dose Testing of Linear Bipolar Circuits," IEEE Trans. Nucl. Sci., Vol. 47, no. 6, pp. 2350-2357, Dec. 2000.

[15] D. M. Hiemstra, "High Total Dose Performance of Various Commercial off the Shelf Operational Amplifiers During Irradiation," IEEE Radiation Effects Data Workshop, Reno (USA), July 2000, pp. 32-38.

[16] M. Menichelli et al., "Total Dose Test of Commercial Off-The-Shelf Components To Be Used in Power Supply for Space Requirements," IEEE Trans. Nucl. Sci., Vol. 47, no. 6, pp. 1879-1884, Dec. 2000.

[17] H. J. Barnaby et al., "Proton Radiation Response Mechanisms in Bipolar Analog Circuits," IEEE Trans. Nucl. Sci., vol. 48, no. 6, pp. 2074-2080, Dec. 2001.

[18] S. T. Gunaseelan, C. R. Selvakumar, and D. Hiemstra, "Radiation Effects and Annealing Behaviour of Operational Amplifiers for Space Application," Proc. of the IEEE Canadian Conference on Electrical and Computer Engineering, Vol. 1, Montreal (Canada), May 4-7 ${ }^{\text {th }}, 2003$, pp. 303-306.

[19] A. H. Johnston and S. M. Guertin, "The Effects of Space Radiation on Linear Integrated Circuits," IEEE Aerospace Conference Proc., Vol. 5, Big Sky (USA), March 18-25 ${ }^{\text {th }}, 2000$, pp. 363-369.

[20] P. C. Adell et al., "Total Dose Effects in a Linear Voltage Regulator," IEEE Trans. Nucl. Sci., vol. 51, no. 6, pp. 3816-3821, Dec. 2004.

[21] J. E. Seiler et al., "Effect of Passivation on the Enhanced Low Dose Rate Sensitivity of National LM124 Operational Amplifiers," IEEE Radiation Effects Data Workshop, Atlanta (USA), July 2004, pp. 42-46.

[22] F. J. Franco et al., "Neutron Effects on Short Circuit Currents of Op Amps and Consequences," IEEE Trans. Nucl. Sci., vol. 52, no. 5, pp. 1530-1537, Oct. 2005.

[23] F. J. Franco, Y. Zong, and J. A. Agapito, "New Details about the Frequency Behavior of Bipolar Op Amps," IEEE Trans. Nucl. Sci., vol. 53, no. 4, pp. 1931-1938, Aug. 2006.

[24] Erric Data Base, http://erric.dasiac.com

[25] A. K. Sharma, K. Sahu, and S. Brashears, "Total Ionizing Dose (TID) Evaluation Results of Low Dose Rate Testing for NASA Programs," IEEE Radiation Effects Data Workshop, Indian Wells (USA), July 1996, pp. 13-18.

[26] M. V. O'Bryan et al. "Radiation Damage and Single Event Effect Results for Candidate Spacecraft Electronics," IEEE Radiation Effects Data Workshop, pp. 106-122, 2000.

[27] GSFC Radiation Data Base, http://radhome.gsfc.nasa.gov

[28] Texas Instruments website, http://www.ti.com

[29] Apex Microtechnologys website, http://www.apexmicrotech.com

[30] J. G. Marques, A. C. Fernandes, I. C. Gonçalves, and A. J. G. Ramalho, "Test Facility at the Portuguese Research Reactor for Irradiations with Fast Neutrons," Proc. of the Workshop on Radiation Effects on Components and Systems, Madrid (Spain), September 2004, pp. 263-265.

[31] J. G. Marques et al., "Radiation tolerant D/A converters for the LHC cryogenic system," Nucl. Inst. and Met. in Phys. Res. Sec. A, Vol. 553, Is. 3, Nov. 2005, pp. 604-612.

[32] ATLAS Standard Radiation Test Methods. Full text available at http://atlas.web.cern.ch/Atlas/GROUPS/FRONTEND/radhard.htm

[33] M. H. Rashid, Microelectronic Circuits : Analysis and Design, Thomson Learning, 1998. 